\title{
Efeito de coberturas mortas vegetais sobre o desempenho da cenoura em cultivo orgânico
}

\author{
Carlos Antonio B Santosi ; Silver R Zandoná ${ }^{1}$; José Antonio A Espindola²; José Guilherme M Guerra ${ }^{2}$; \\ Raul de Lucena D Ribeiro ${ }^{1}$ \\ ${ }^{1}$ UFRuralRJ, Ladeira da Meteorologia, 03, 23890-000 Seropédica-RJ; ${ }^{2}$ Embrapa Agrobiologia, C. Postal 74505, 23890-000 Seropédica- \\ RJ; agbarreto@ufrrj.br, srzandona@yahoo.com.br; gmguerra@cnpab.embrapa.br; jose@cnpab.embrapa.br
}

\begin{abstract}
RESUMO
Avaliou-se o efeito de coberturas mortas com resíduos de diferentes espécies de plantas no rendimento da cenoura em cultivo orgânico e nos níveis de reinfestação pela vegetação espontânea. $\mathrm{O}$ experimento foi conduzido no Sistema Integrado de Produção Agroecológica ("Fazendinha Agroecológica Km 47”), em Seropédica-RJ, empregando delineamento de blocos casualizados, com quatro tratamentos e seis repetições. Os tratamentos consistiram de coberturas dos canteiros com a biomassa aérea, seca e triturada, de capim Cameroon (Pennisetum purpureum), gliricídia (Gliricidia sepium) e guandu (Cajanus cajan), além da testemunha (sem qualquer cobertura). Por ocasião da colheita da cenoura, determinou-se a produtividade e as médias em peso, comprimento e diâmetro máximo das raízes tuberosas. A reinfestação de ervas espontâneas foi estimada pelo número total de indivíduos por $\mathrm{m}^{2}$, sendo as coletas realizadas aos 20,50 e 80 dias após a semeadura. A cenoura cultivada nas parcelas tratadas com resíduos de leguminosas apresentou aumentos significativos de todas as variáveis fitotécnicas, com exceção para o comprimento médio da raiz. Nas parcelas cobertas com resíduos da gramínea, os resultados foram estatisticamente iguais aos da testemunha. A reinfestação por plantas espontâneas por unidade de área cultivada alcançou níveis da ordem de $300 \%$ superiores em parcelas sem cobertura do solo, na comparação com aquelas que receberam as palhadas.
\end{abstract}

Palavras-chave: Daucus carota, Pennisetum purpureum, Gliricidia sepium, Cajanus cajan, ervas espontâneas.

\begin{abstract}
Effect of mulch of different plant species on the performance of organically grown carrot

The effect of soil mulch with different plant species was evaluated in relation to the performance of organically grown carrots and to weed population levels. The experiment was carried out at the Integrated Agroecological Production System located in Seropédica, Metropolitan Region of Rio de Janeiro State (Baixada Fluminense), Brazil, using a randomized blocks design comprising four treatments replicated six times. These treatments consisted of soil mulch with chopped and dried above-ground biomass of Cameroon grass (Pennisetum purpureum), gliricidia (Gliricidia sepium), and pigeon pea (Cajanus cajan) compared to uncovered plots (control treatment). Carrot marketable yield and root characteristics (weight, length, and major diameter) were determined at harvesting. Reinfestation by weeds was estimated through the total number of individuals per square meter of cultivated area and the assessment carried out at 20, 50 and 80 days after sowing. Carrot (cv. Brasília) showed significantly increased values regarding all the variables (except for root length) in the plots treated with legume amendments (pigeon pea and gliricidia). As for the Cameroon grass mulch, results were statistically equivalent to the control treatment. Weed populations by cultivated area unit reached levels up to $300 \%$ higher in the non-mulch plots compared to the ones covered with plant residues.
\end{abstract}

Keywords: Daucus carota, Pennisetum purpureum, Gliricidia sepium, Cajanus cajan, weeds.

\section{(Recebido para publicação em15 de julho de 2009; aceito em 7 de dezembro de 2010) (Received on July 15, 2009; accepted on December 7, 2010)}

\begin{abstract}
A cenoura (Daucus carota) apresentase como uma das hortaliças mais procuradas no mercado de alimentos orgânicos do Rio de Janeiro. No entanto, observa-se, com freqüência, uma reduzida oferta desta olerícola, o que é, sobretudo atribuído a dificuldades tecnológicas, devido a limitações inerentes à obtenção de raízes de melhor padrão comercial, respeitando as normas do MAPA (2008).

No modelo convencional, de base agroquímica, as áreas cultivadas com cenoura têm seu manejo facilitado pelo emprego sistemático de herbicidas de
\end{abstract}

pré e pós-emergência, o que, na maioria das vezes, dispensa capinas durante o ciclo desta hortaliça. Nos sistemas orgânicos de produção torna-se necessária maior utilização de mão-de-obra, visto que o controle de plantas espontâneas na lavoura é realizado manualmente, acarretando elevação dos preços praticados na comercialização da cenoura. Nesse contexto, o uso de coberturas mortas vegetais aparece como uma técnica capaz de amenizar tal limitação, por manter as populações reinfestantes em níveis toleráveis nasáreas cultivadas (Maclean et al., 2003; Oliveira et al., 2008). Manter e melhorar a fertilidade do solo são metas também favorecidas pelo uso de coberturas mortas (Espindola et al., 2006).

A disponibilização de nutrientes contidos nos resíduos vegetais normalmente varia com a espécie de planta empregada para cobertura morta do solo. Kumar \& Goh (2002), por exemplo, obtiveram maior produtividade do trigo quando cultivado em solo coberto com resíduos de leguminosas do que com resíduos de gramíneas. Esse resultado foi atribuído à elevada relação $\mathrm{C} / \mathrm{N}$ das gramíneas, capaz de imobilizar o nitrogênio do solo. 
A palhada oriunda da biomassa aérea de leguminosas fixadoras de $\mathrm{N}_{2}$, fragmentada e depositada na superfície do solo, é rica em nutrientes, os quais são prontamente liberados devido à sua acelerada taxa de decomposição (Aita \& Giacomini, 2003), influindo positivamente no desempenho agronômico das culturas. Isto foi evidenciado nos resultados encontrados por Oliveira et al. (2008), os quais constataram produtividade e acumulação de nitrogênio mais altas em alface cultivada com cobertura morta formada de palhas de leguminosas, quando comparadas com palhas de gramíneas.

Além dos benefícios já mencionados, a cobertura morta promove melhorias na agregação do solo (Corrêa, 2002), previne processos erosivos (Smolikowski et al., 2001) e reduz a necessidade de irrigação (Lima et al., 2009).

O presente estudo teve por objetivo comparar o efeito de diferentes coberturas mortas do solo, no rendimento da cenoura em cultivo orgânico, assim como no nível de reinfestação pela vegetação espontânea durante o ciclo da hortaliça, sob as condições edafoclimáticas da Baixada Fluminense.

\section{MATERIAL E MÉTODOS}

O experimento foi conduzido de agosto a novembro de 2006, no Sistema Integrado de Produção Agroecológica (SIPA, "Fazendinha Agroecológica Km 47”), localizado no município de Seropédica, Região Metropolitana do estado do Rio de Janeiro (Baixada Fluminense). $\mathrm{O}$ clima da região pertence à classe Aw, segundo Köppen, apresentando inverno pouco rigoroso e seco, com chuvas freqüentes e temperaturas muito elevadas durante o verão (Figura 1). O solo da área experimental é classificado como Argissolo Vermelho-Amarelo (Embrapa, 2006), cuja análise química (camada de $0-20 \mathrm{~cm}$ ), efetuada de acordo com metodologia preconizada por Silva (1999), forneceu os seguintes resultados: $\mathrm{pH}$ (em água) $=5,3 ; \mathrm{Al}^{+++}=$ $0,0 \mathrm{cmol}_{\mathrm{c}} \mathrm{dm}^{-3} ; \mathrm{Ca}^{++}=3,9 \mathrm{cmol}_{\mathrm{c}} \mathrm{dm}^{-3}$; $\mathrm{Mg}^{++}=1,1 \mathrm{cmol}_{\mathrm{c}} \mathrm{dm}^{-3}$; $\mathrm{P}$ disponível= 135,0 $\mathrm{mg} \mathrm{dm}^{-3} ; \mathrm{K}^{+}=168,2 \mathrm{mg} \mathrm{dm}^{-3}$.

O delineamento experimental adota- do foi de blocos casualizados, com seis repetições. Os tratamentos consistiram da cobertura morta do solo usando as fontes de resíduos vegetais: capim Cameroon (Pennisetum purpureum), gliricídia (Gliricidia sepium) e guandu (Cajanus cajan). Na testemunha não se empregou qualquer tipo de cobertura do solo. Os resíduos empregados foram provenientes do corte da biomassa aérea do guandu e do capim Cameroon, e de folhas e ramos finos originados da poda da leguminosa arbórea gliricídia. Esses materiais foram triturados e, em seguida, secos à sombra. A quantidade aplicada de cada cobertura morta (em base seca) totalizou 2,5 $\mathrm{kg} \mathrm{m}^{-2}$ de canteiro, tomando por referência, resultado de pesquisa similar anteriormente conduzida na mesma localidade (Oliveira et al., 2008). A área do experimento foi preparada por meio de moto-encanteirador, com cada parcela ocupando uma área de $2,0 \mathrm{~m}^{2} \mathrm{de}$ canteiro $(2,0 \times 1,0 \mathrm{~m})$.

Antecedendo a semeadura da cenoura, realizou-se uma adubação orgânica com esterco bovino curtido na dose única de 8,0 tha-1 (base seca), equivalendo a $100 \mathrm{~kg}$ de $\mathrm{N} \mathrm{ha}^{-1}$. A cultivar Brasília foi semeada em sulcos espaçados de 25 cm no sentido da largura dos canteiros. Foram realizados dois desbastes, sendo o primeiro aos 14 dias após a semeadura, conservando-se entre 30 e 40 plântulas por metro, e o segundo aos 23 dias, assegurando-se um estande final de 20 plantas por metro de sulco, conforme indicado para a cultura da cenoura (Filgueira, 2000). Utilizaram-se colmos de bambu, longitudinalmente seccionados e dispostos sobre cada sulco semeado, visando evitar que os resíduos vegetais distribuídos na superfície dos canteiros pudessem interferir na germinação da cenoura. Os colmos de bambu foram retirados dos canteiros quatro dias após a semeadura da hortaliça.

Por ocasião da implantação do experimento, foram coletadas amostras de cada tipo de cobertura morta para determinação dos teores de nutrientes. Essas amostras foram mantidas em estufa com ventilação forçada e regulada a $65^{\circ} \mathrm{C}$, onde permaneceram até atingir peso constante, sendo então moídas. A determinação do teor de $\mathrm{N}$ contido na fitomassa seca baseou-se no método de
Bremner \& Mulvaney (1982). O teor de $\mathrm{P}$ foi determinado por colorimetria, através da formação de cor azul do complexo fosfato-molibdato em presença de ácido ascórbico; e do K, por espectrofotometria de absorção atômica (Embrapa, 1997). As análises relativas a $\mathrm{Ca}$ e $\mathrm{Mg}$ foram também efetuadas por espectrofotometria, após digestão nítrico-perclórica (Bataglia et al., 1983).

As populações reinfestantes de ervas espontâneas foram avaliadas, somandose o número total de indivíduos por $\mathrm{m}^{2}$ de canteiro, em três épocas distintas (20, 50 e 80 dias) a contar da semeadura da cenoura. Essas amostragens foram padronizadas por meio do uso de moldura de madeira, representando um quadrado com área interna de $0,25 \mathrm{~m}^{2}$, dispostas ao acaso na área útil de cada parcela. Após cada avaliação, realizou-se capina manual das parcelas.

A colheita da cenoura foi realizada 90 dias após a semeadura, agrupando-se as plantas contidas em área centralizada de $0,8 \mathrm{~m}^{2}$ por parcela experimental. Foram avaliados: peso, comprimento e diâmetro máximo das raízes tuberosas, tomando por base as normas de classificação desta hortaliça sugerida por Hortibrasil (2002), assim como a produtividade da cultura. Para este último parâmetro, foram consideradas apenas as cenouras com diâmetro e comprimento acima de 2,5 e 12,0 cm, respectivamente, adotando-se, assim, os padrões comerciais estabelecidos por Silva et al. (1991). Na mesma data, foram também obtidas amostras das raízes e da parte aérea para determinação dos teores de nutrientes, obedecendo à metodologia já citada.

Os dados obtidos foram submetidos à análise de variância através do teste $\mathrm{F}$. Para comparação das médias foi aplicado o teste de Scott-Knott $(\mathrm{p}<0,05)$ na comparação das respectivas médias, utilizando o software SISVAR versão 4.3.

\section{RESULTADOS E DISCUSSÃO}

Os resíduos de gliricídia apresentaram teores mais altos de $\mathrm{N}, \mathrm{P}, \mathrm{K}, \mathrm{Ca}$ e $\mathrm{Mg}$, enquanto os de guandu mostraram maiores concentrações de N, P e Ca em 


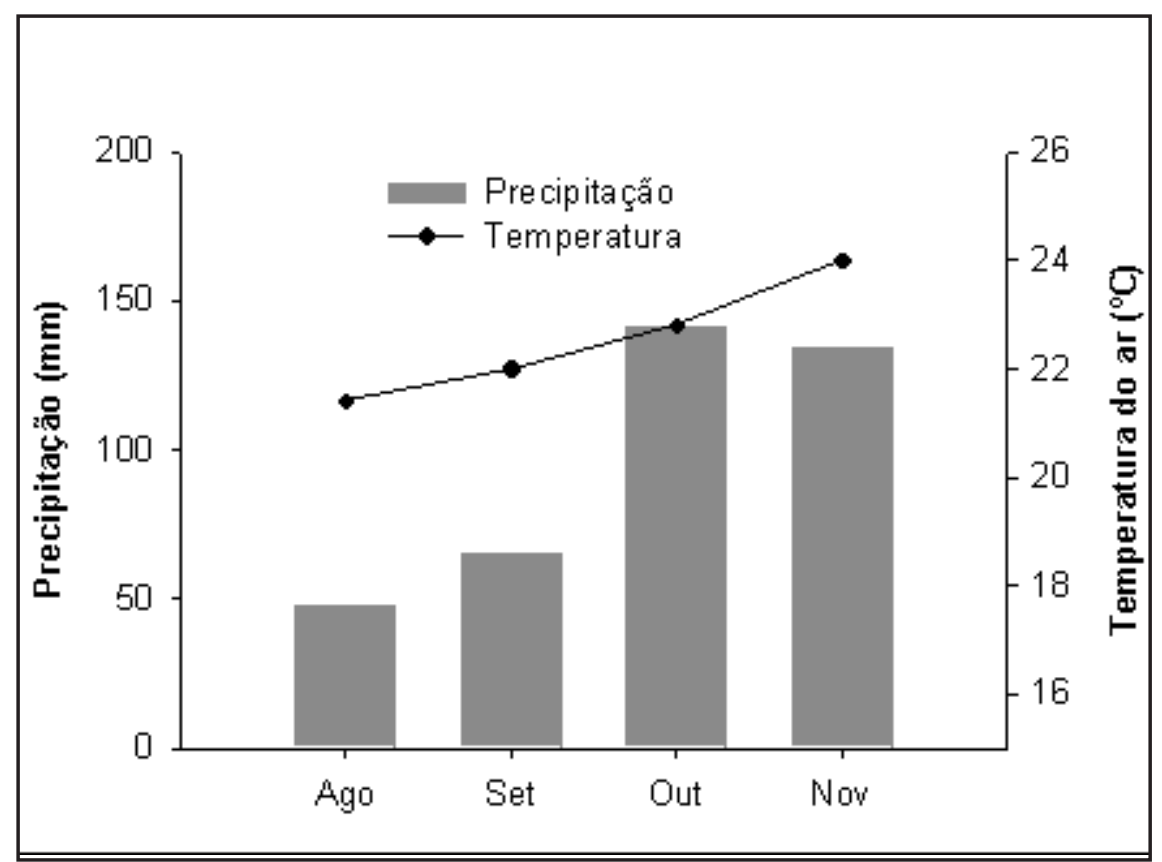

Figura 1. Variações mensais da temperatura média do $\operatorname{ar}\left({ }^{\circ} \mathrm{C}\right)$ e da precipitação pluviométrica $(\mathrm{mm})$, referentes ao período experimental, na Baixada Fluminense (monthly variations of average air temperature $\left({ }^{\circ} \mathrm{C}\right)$ and rainfall $(\mathrm{mm})$ during the experimental period, at Baixada Fluminense). Seropédica, Pesagro, 2006.

Tabela 1. Médias dos teores de macronutrientes na biomassa seca de diferentes espécies vegetais usada para cobertura morta de cenoura (cv. 'Brasília') em sistema orgânico de cultivo (averages of macronutrients contents of different vegetal species dry matter used for carrot ('Brasília') mulching in organic farming system. Seropédica, Pesagro, 2006.

\begin{tabular}{lrrrrr}
\hline \multirow{2}{*}{ Espécie } & \multicolumn{1}{c}{$\mathbf{N}$} & $\mathbf{P}$ & $\mathbf{K}$ & $\mathbf{C a}$ & $\mathbf{M g}$ \\
\cline { 2 - 6 } & 27,28 & 2,81 & \multicolumn{5}{c}{8,30} & 25,50 & 3,45 \\
\hline Guandu (Cajanus cajan) $^{1}$ & 32,03 & 2,45 & 19,60 & 35,60 & 4,95 \\
Gliricídia (Gliricidia sepium) & 4,32 & 0,95 & 12,30 & 6,40 & 3,75 \\
Cameroon (Pennisetum purpureum)
\end{tabular}

${ }^{1}$ Resíduos secos e triturados: guandu e Cameroon = biomassa aérea roçada; gliricídia = folhas e ramos finos provenientes de poda (dried and chopped residues: pigeon pea and Cameroon = chopped aboveground part biomass; gliricidia = leaves and thin branches obtained from pruning).

relação ao capim Cameroon (Tabela 1).

A produtividade da cenoura nos tratamentos com cobertura morta de resíduos de leguminosas mostrou-se superior à do tratamento com o capim Cameroon e ao tratamento-testemunha (Tabela 2). Nas parcelas cobertas com guandu e gliricídia, a produtividade de cenoura apresentou incrementos de, respectivamente, 24,3 e $17,5 \%$, na comparação com as parcelas sem utilização de cobertura do solo.

$\mathrm{O}$ efeito benéfico das coberturas mortas com resíduos de leguminosas deve-se, provavelmente, aos nutrientes os valores mais altos também foram acarretados pelas coberturas mortas de gliricídia e de guandu (Tabela 2). Por outro lado, não houve diferenças significativas entre tratamentos com relação ao comprimento das raízes colhidas. Possivelmente, a uniformidade no padrão de desenvolvimento das raízes tuberosas está associada às características genéticas da cultivar utilizada.

Com respeito à massa seca da parte aérea e das raízes de cenoura, os maiores valores, mais uma vez, corresponderam aos tratamentos com resíduos provenientes das leguminosas (Tabela 2).

Quanto aos teores de nutrientes acumulados na cenoura (Tabela 3), com exceção de $\mathrm{K}$ e $\mathrm{Mg}$ na parte aérea e de $\mathrm{P}$ e $\mathrm{Mg}$ nas raízes, foram detectadas diferenças significativas entre tratamentos. Na parte aérea dessa hortaliça, destacouse a cobertura do solo com resíduos de capim Cameroon, a qual proporcionou aumentos significativos dos teores de $\mathrm{P}$ e $\mathrm{Ca}$ nos tecidos. Em contrapartida, o maior acúmulo de $\mathrm{N}$ ocorreu em resposta às coberturas de gliricídia e de guandu. Em relação aos níveis de nutrientes nas raízes, os tratamentos com as leguminosas proporcionaram aumentos significativos dos teores de $\mathrm{N}$, K e Ca quando comparados aos demais tratamentos. Os resíduos das leguminosas guandu e gliricídia (Tabela 1) apresentam teores mais altos destes nutrientes, com exceção do K contido no resíduo de guandu, o que pode explicar esse comportamento dos nutrientes na planta de cenoura.

A reinfestação pelas ervas espontâneas na área experimental foi reduzida de forma expressiva pelas coberturas mortas do solo, independentemente do tipo de resíduo vegetal utilizado (Figura 2). Durante o cultivo da cenoura, a densidade populacional da vegetação espontânea alcançou níveis da ordem de $300 \%$ superiores nas parcelas do tratamento-testemunha, o que influiu negativamente no desempenho da hortaliça devido a competições por nutrientes e água. As espécies de ervas, identificadas de acordo com Lorenzi (2000), foram: caruru (Amaranthus lividus), capim colonião (Panicum maximum), capim colchão (Digitaria horizontalis), falsaserralha (Emilia sonchifolia), pé-de- 
Tabela 2. Peso fresco, diâmetro máximo, comprimento, e produtividade da cenoura 'Brasília' produzida em cultivo orgânico com diferentes coberturas do solo com resíduos vegetais (fresh weight, maximum diameter, root length and yield of organically grown carrot 'Brasília' under different plant species residues used for mulch). Seropédica, Pesagro, 2006.

\begin{tabular}{lcccc}
\hline Cobertura morta & \multicolumn{1}{c}{$\begin{array}{c}\text { Peso } \\
\text { (g) }\end{array}$} & $\begin{array}{c}\text { Diâmetro Comprimento Produtividade } \\
\text { (cm) }\end{array}$ & $\begin{array}{c}\text { (cm) } \\
\left(\mathbf{t ~ h a}^{-1}\right)\end{array}$ \\
\hline Cameroon (P. purpureum) & $53,51 \mathrm{~b}$ & $3,1 \mathrm{~b}$ & $14,0 \mathrm{a}$ & $31,24 \mathrm{~b}$ \\
Guandu (C. cajan) & $60,74 \mathrm{a}$ & $3,4 \mathrm{a}$ & $14,0 \mathrm{a}$ & $36,64 \mathrm{a}$ \\
Gliricídia (G. sepium) & $59,40 \mathrm{a}$ & $3,4 \mathrm{a}$ & $14,0 \mathrm{a}$ & $34,63 \mathrm{a}$ \\
Testemunha & $53,47^{2} \mathrm{~b}$ & $3,2 \mathrm{~b}$ & $13,0 \mathrm{a}$ & $29,48 \mathrm{~b}$ \\
\hline CV $(\%)$ & 7,79 & 3,89 & 3,52 & 8,31 \\
\hline
\end{tabular}

${ }^{1}$ Resíduos secos e triturados: guandu e Cameroon = biomassa aérea roçada; gliricídia $=$ folhas e ramos finos procedentes de poda (dried and chopped residues: pigeon pea and Cameroon $=$ chopped aboveground part biomass; gliricidia = leaves and thin branches obtained from pruning); ${ }^{2} \mathrm{Os}$ valores representam médias de seis repetições; médias seguidas da mesma letra em cada coluna não diferem entre si pelo teste de Scott-Knott $(\mathrm{p}<0,05)($ values represent means of six replications; means followed by the same letter in each column do not differ by the Scott-Knott test $(\mathrm{p}<0.05))$.

galinha (Eleusine indica), quebra-pedra (Phillanthus corcovadensis), tiririca (Cyperus sp.) e trapoeraba (Commelina benghalensis).

Variados mecanismos podem estar relacionados à eficácia do controle da população de ervas espontâneas pelas coberturas mortas. Incluem-se principalmente alterações na incidência de luz e temperatura, que afetam as taxas de germinação do banco de sementes (Ma- cenoura.

O estudo evidenciou efeitos benéficos do uso de coberturas mortas do solo, especialmente com resíduos de leguminosas (gliricídia e guandu), no cultivo orgânico da cenoura 'Brasília'. Essa prática cultural propiciou nas condições edafoclimáticas da Baixada Fluminense, aumentos significativos da produtividade, melhoria de aspectos comerciais e aumento dos teores de nitrogênio, potássio e cálcio das raízes de cenoura. Independentemente do resíduo utilizado como cobertura morta do solo, estes reduziram eficazmente a reinfestação de plantas espontâneas presentes na área.

\section{AGRADECIMENTOS}

Os autores agradecem o apoio da FAPERJ na realização deste estudo.

\section{REFERÊNCIAS}

plantas, como já relatado por Constantin (2001). Não se deve descartar, ainda, uma possível contribuição de compostos inibidores, conhecidos genericamente como aleloquímicos (Pires \& Oliveira, 2001), porventura presentes nos resíduos utilizados nos canteiros de
AITA C; GIACOMINI SJ. 2003. Decomposição e liberação de nitrogênio de resíduos culturais de plantas de cobertura de solo solteiras e consorciadas. Revista Brasileira de Ciência do Solo 27: 601-612.
BATAGLIA OC; FURLANI AMC; TEIXEIRA

Tabela 3. Médias da massa seca e dos teores de nutrientes da parte aérea e das raízes de cenoura 'Brasília' produzida em cultivo orgânico com diferentes coberturas mortas vegetais (average shoot and root dry matter and nutrient content of organically grown carrot 'Brasília' under different plant species residues used for mulch). Seropédica, Pesagro, 2006.

\begin{tabular}{|c|c|c|c|c|c|c|}
\hline \multirow{2}{*}{ Cobertura morta ${ }^{1}$} & \multirow{2}{*}{$\begin{array}{c}\text { Massa seca } \\
\left(\mathrm{t} \mathrm{ha}^{-1}\right)\end{array}$} & \multicolumn{5}{|c|}{ Teor de nutriente $\left(\mathrm{g} \mathrm{kg}^{-1}\right)$} \\
\hline & & $\mathbf{N}$ & $\mathbf{P}$ & $\mathbf{K}$ & $\mathbf{C a}$ & $\mathrm{Mg}$ \\
\hline & \multicolumn{6}{|c|}{ Parte aérea } \\
\hline Cameroon (P. purpureum) & $2,05 \mathrm{~b}$ & $14,33 \mathrm{c}$ & $2,63 \mathrm{a}$ & $25,04 \mathrm{a}$ & $19,85 \mathrm{a}$ & $1,84 \mathrm{a}$ \\
\hline Guandu (C. cajan) & $2,83 \mathrm{a}$ & $19,17 \mathrm{~b}$ & $2,45 \mathrm{~b}$ & $23,63 \mathrm{a}$ & $16,55 \mathrm{~b}$ & $1,80 \mathrm{a}$ \\
\hline Gliricídia (G. sepium) & $2,67 \mathrm{a}$ & $22,83 \mathrm{a}$ & $2,11 \mathrm{c}$ & $24,54 \mathrm{a}$ & $16,84 \mathrm{~b}$ & $1,82 \mathrm{a}$ \\
\hline Testemunha (sem cobertura) & $2,19 b^{2}$ & $15,50 \mathrm{c}$ & $2,19 \mathrm{c}$ & $24,38 \mathrm{a}$ & $18,13 \mathrm{~b}$ & $1,82 \mathrm{a}$ \\
\hline \multirow[t]{2}{*}{ CV (\%) } & 17,27 & 8,66 & 6,07 & 15,93 & 10,21 & 4,11 \\
\hline & \multicolumn{6}{|c|}{ Raíz } \\
\hline Cameroon (P. purpureum) & $2,59 \mathrm{~b}$ & $12,80 \mathrm{~d}$ & $2,80 \mathrm{a}$ & $30,95 \mathrm{~b}$ & $3,67 \mathrm{~b}$ & $1,65 \mathrm{a}$ \\
\hline Guandu (C. cajan) & $2,81 \mathrm{a}$ & $18,73 \mathrm{~b}$ & $2,77 \mathrm{a}$ & $35,00 \mathrm{a}$ & $4,44 \mathrm{a}$ & $1,59 \mathrm{a}$ \\
\hline Gliricídia (G. sepium) & $3,09 \mathrm{a}$ & $21,60 \mathrm{a}$ & $2,75 \mathrm{a}$ & $37,20 \mathrm{a}$ & $4,25 \mathrm{a}$ & $1,64 \mathrm{a}$ \\
\hline Testemunha (sem cobertura) & $2,33 \mathrm{~b}$ & $15,52 \mathrm{c}$ & $2,66 \mathrm{a}$ & $31,25 \mathrm{~b}$ & $3,43 \mathrm{~b}$ & $1,63 \mathrm{a}$ \\
\hline CV (\%) & 14,79 & 12,08 & 5,67 & 9,64 & 6,67 & 4,09 \\
\hline
\end{tabular}

${ }^{1}$ Resíduos secos e triturados: guandu e Cameroon = biomassa aérea roçada; gliricídia = folhas e ramos finos procedentes de poda $($ dried and chopped residues: pigeon pea and Cameroon = chopped aboveground part biomass; gliricidia $=$ leaves and thin branches obtained from pruning); ${ }^{2}$ Os valores representam médias de seis repetições; médias seguidas da mesma letra em cada coluna não diferem entre si pelo teste de Scott-Knott $(\mathrm{p}<0,05)$ (values are means of six replications; means followed by the same letter in each column do not differ by the Scott-Knott test $(\mathrm{p}<0.05))$. 


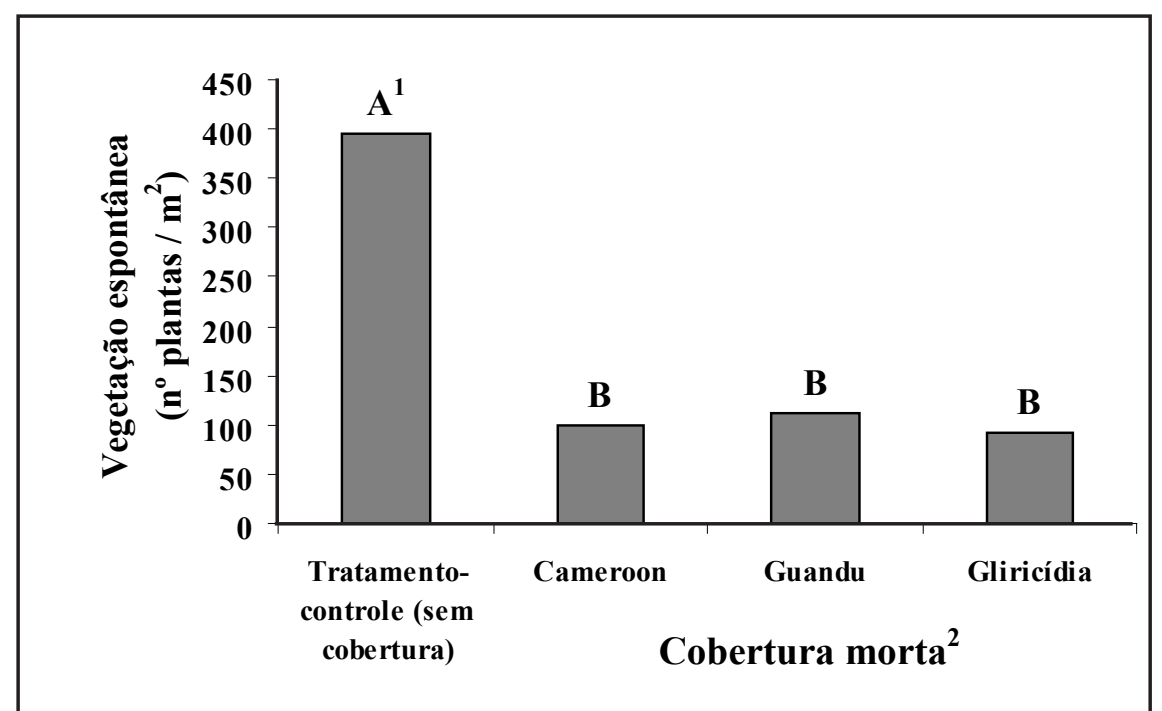

Figura 2. Níveis de reinfestação pelas ervas espontâneas em cultivo orgânico de cenoura 'Brasília', com diferentes coberturas mortas vegetais (levels of weed reinfestation in organically grown carrot 'Brasília', with different plants used for mulch). Seropédica, Pesagro, 2006. ${ }^{1}$ Os valores representam médias de seis repetições; letras iguais em cada coluna indicam médias que não diferem pelo teste de Scott-Knott $(\mathrm{p}<0,05)$ (values represent means of six replications; means followed by the same letter in each column do not differ by the ScottKnott test $(\mathrm{p}<0.05)) ;{ }^{2}$ Material seco e triturado: guandu e capim Cameroon = biomassa aérea roçada; gliricídia $=$ folhas e ramos finos procedentes de poda (dried and chopped residues: pigeon pea and Cameroon $=$ chopped aerial biomass; gliricidia $=$ leaves and thin branches obtained from pruning).

JPF; GALLO JR. 1983. Métodos de análise química de plantas. Campinas: Instituto Agronômico, 48p. (Instituto Agronômico. Boletim, 78).

BREMNER JM; MULVANEY CS. 1982. Nitrogen total. In: PAGE AL. (ed.). Methods of soil analysis. 2. ed. Madison: SSSA, Part 2. p. 595-624.

CONSTANTIN J. 2001. Métodos de manejo. In: OLIVEIRA JÚNIOR RS; CONSTANTIN J. (coord). Plantas daninhas e seu manejo. Guaíba: Editora Agropecuária, p. 103-144.

CORRÊA JC. 2002. Efeito de sistemas de cultivo na estabilidade de agregados de um Latossolo Vermelho-Amarelo em Querência, MT. Pesquisa Agropecuária Brasileira 37: 203-209.

EMBRAPA. 2006. Centro Nacional de Pesquisa de Solos. Sistema brasileiro de classificação de solo. 2 ed. Rio de Janeiro,306p.

EMBRAPA. 1997. Centro Nacional de Pesquisa de Solos. Manual de métodos de análise de solo. Rio de Janeiro, 212 p.
LIMA ME; CARVALHO DF; SOUZA AP; GUERRA JGM; RIBEIRO RLD. 2009. Desempenho da alface em cultivo orgânico com e sem cobertura morta e diferentes lâminas d'água. Ciência e Agrotecnologia 33: 1503-1510.

MACLEAN RH; LITSINGER JA; MOODY K; WATSON AK; LIBETARIO EM. 2003. Impact of Gliricidia sepium and Cassia spectabilis hedgerows on weeds and insect pests of upland rice. Agriculture, Ecosystems and Environment 94: 275-288.

MAPA. 2008. (Ministério da Agricultura, Pecuária e Abastecimento). Instrução Normativa 64. Disponível em: http://extranet.agricultura.gov. br/ Acesso em: 02 de fev. de 2009.

MATEUS GP; CRUSCIOL CAC; NEGRISO E. 2004. Palhada do sorgo de guiné gigante no estabelecimento de plantas daninhas em área de plantio direto. Pesquisa Agropecuária Brasileira 39: 539-542.

OLIVEIRA FF; GUERRA JGM; ALMEIDA DL; RIBEIRO RLD; ESPINDOLA JAA; RICCI MSF; CEDDIA MB. 2008. Avaliação de coberturas mortas em cultura de alface sob manejo orgânico. Horticultura Brasileira 26: 216-220.

PIRES NM; OLIVEIRA VR. 2001. Alelopatia. In: OLIVEIRA JÚNIOR RS; CONSTANTIN J. (coord). Plantas daninhas e seu manejo. Guaíba: Editora Agropecuária, p. 145-185.

PÖTTKER D; ROMAN ES. 1994. Efeito de resíduos de culturas e do pousio de inverno sobre a resposta do milho a nitrogênio. Pesquisa Agropecuária Brasileira 29: $763-$ 770.

RESENDE FV; SOUZA LS; OLIVEIRA PSR; GUALBERTO R. 2005. Uso de cobertura morta vegetal no controle da umidade e temperatura do solo, na incidência de plantas invasoras e na produção da cenoura em cultivo de verão. Ciência e Agrotecnologia 29: 100-105.

SILVA FC. 1999. Manual de análises químicas de solos, plantas e fertilizantes. Embrapa Comunicação para Transferência de Tecnologia, Brasília, 370p.

SILVA JLO; CALBO AG; HENZ GP. 1991. Classificação e beneficiamento de hortaliças. Informe Agropecuário 15: 48-53.

SMOLIKOWSKI B; PUIG H; ROOSE E. 2001. Influence of soil protection techniques on runoff, erosion and plant production on semiarid hillsides of Cabo Verde. Agriculture, Ecosystems and Environment 87: 67-80. 